Check for updates

Cite this: Soft Matter, 2020,

16, 8101

Received 22nd May 2020,

Accepted 18th August 2020

DOI: $10.1039 / \mathrm{d} 0 \mathrm{sm} 00954 \mathrm{~g}$

rsc.li/soft-matter-journal

\title{
Self-assembly behavior of experimentally realizable lobed patchy particles $\uparrow$
}

\author{
Sanjib Paul (D) and Harish Vashisth (D) *
}

\begin{abstract}
We report simulation studies on the self-assembly behavior of five different types of lobed patchy particles of different shapes (snowman, dumbbell, trigonal planar, square planar, and tetrahedral). Inspired by an experimental method of synthesizing patchy particles (Wang et al., Nature, 2012, 491, 51-55), we control the lobe size indirectly by gradually varying the seed diameter and study its effect on self-assembled structures at different temperatures. Snowman shaped particles self-assemble only at a lower temperature and form two-dimensional sheets, elongated micelles, and spherical micelles, depending on the seed diameter. Each of the four other lobed particles self-assemble into four distinct morphologies (random aggregates, spherical aggregates, liquid droplets, and crystalline structures) for a given lobe size and temperature. We observed temperature-dependent transitions between two morphologies depending on the type of the lobed particle. The self-assembled structures formed by these four types of particles are porous. We show that their porosities can be tuned by controlling the lobe size and temperature.
\end{abstract}

Self-assembly of colloidal patchy particles is an emerging and novel way of obtaining unique morphologies for various technological and biomedical applications. ${ }^{1-6}$ Guided by the site-specific directional interactions, these particles have the potential to self-assemble into higher order structures (for example, chains, ${ }^{7}$ two-dimensional sheets, ${ }^{7}$ and even more complex structures like a diamond crystal $^{8}$ ) which cannot be obtained by other synthetic routes. Because of their unparalleled importance, recent years have witnessed an effort, both in theory and experiments, in designing patchy particles, and studying their self-assembly behavior. ${ }^{9-22}$ Among key factors that control the phases and the morphologies of self-assembled structures are the size of the patches, interaction strength

Department of Chemical Engineering, University of New Hampshire, 33 Academic Way, Durham, NH 03824, USA. E-mail: harish.vashisth@unh.edu

$\dagger$ Electronic supplementary information (ESI) available: Thirteen figures (modeling of snowman shaped particles, simulation domains, radial distribution functions, and pore size distributions) and simulation methods are included. See DOI: 10.1039/d0sm00954g between the patches, temperature and volume fraction of particles along with the intrinsic factors like the number and the location of patches.

In a recent study, ${ }^{23}$ spherical Janus particles, which interacted through DNA functionalized attractive patches, were found to self-assemble into diverse structures (colloidal micelles, chains, and bilayers) depending on the patch size. Patch size is a critical property that rules the self-assembly of particles into desired structures. While larger patches allow more flexibility and thus lead to misassembly, smaller patches are more likely to produce targeted structures. ${ }^{24,25}$ Liu et al. ${ }^{26}$ showed that the dumbbell shaped $\mathrm{Au}-\mathrm{Fe}_{3} \mathrm{O}_{4}$ nanocrystals comprised two spherical beads (the particles having two spherical beads are called snowman shaped particles in the present study), in which one bead is attractive and another one is repulsive, self-assemble into different superstructures (dimers, trimers, tetramers) at different bead size ratios. Wang et al. ${ }^{11}$ reported a method of making non-spherical patchy particles, where patches around a central spherical seed appear as protrusions or lobes, and showed a way of getting different self-assembled structures by controlling the patch size. Using the emulsion and evaporation technique, ${ }^{27}$ they first made clusters of different shapes (spheres, dumbbells, triangles, tetrahedral, triangular bipyramidal, octahedral, and pentagonal bipyramidal) which were composed of $n$ ( $n$ varies from 1 to 7) number of amidinated polystyrene beads. In the next step, the clusters were swelled and polymerized using the styrene monomers. The exposed parts of the polystyrene beads, after the swelling and polymerization are carried out, appear as protrusions or lobes and act as patches. Patch sizes were observed to depend entirely on the extent of swelling. Higher is the swelling, lower is the patch size and vice versa. The amidinated patches can be subjected to further functionalization by molecules like complementary DNA strands or pilandromes to induce directional interactions between the particles. The patchy particles thus produced self-assemble and generate different morphological structures depending upon their patch sizes. For example, particles having two 
patches on two opposite sides of the central seed self-assemble into linear chains, when the patch size is smaller. But when patches are made bigger, the same particles were observed to form branched chains.

In our previous work, ${ }^{28}$ we studied the self-assembly of seven different types of lobed particles (snowman, dumbbell, trigonal planar, square planar, tetrahedral, trigonal bipyramidal, and octahedral). We kept the seed to lobe diameter ratio constant and varied the attractive interactions between the lobes to understand its effect on the self-assembly. The selfassembled structures generated by these particles (except snowman) were found to be either porous amorphous or porous crystalline. A porous self-assembled structure could find many applications, for example, in tissue engineering, if the pore diameters are in the micron scale range. ${ }^{29,30}$ The dumbbell, trigonal planar, and square planar particles were observed to generate highly porous structures compared to the other three types of particles (tetrahedral, trigonal bipyramidal, and octahedral). ${ }^{28}$ The self-assembled morphologies and the porosities were found to be independent of a range of volume fractions of the lobed particles.

In the present work, we extended our previous study, and investigated in detail the effect of lobe size on the selfassembled morphologies and porosities for five different particles (snowman, dumbbell, trigonal planar, square planar, and tetrahedral). Except for the square planar particles, the other four types of particles are experimentally realizable. ${ }^{11,31-33}$ The square planar lobed particles are analogues of the square planar patchy particles (having four surface patches at four equatorial positions) studied by Zhang et $a .^{7}{ }^{7}$ The inclusion of square planar particles in the present study allows the examination of the effect of shapes on the self-assembled structures formed by two different types of particles having the same number of lobes (square planar and tetrahedral). To model the lobed particles, we followed an approach inspired by the work of Pine and coworkers. ${ }^{11}$ We have not chosen trigonal bipyramidal and octahedral lobed particles in this study, as these two types of particles are not promising to produce highly porous structures, as revealed by our previous work. ${ }^{28}$ Moreover, the porosities generated by these two types of particles were found to be comparable with the tetrahedral lobed particles and therefore, in this work, we choose to study only the tetrahedral particles among the tetrahedral, trigonal bipyramidal, and octahedral lobed particles.

Specifically, we studied the self-assembly of five different types of particles: (i) snowman, (ii) dumbbell, (iii) trigonal planar, (iv) square planar, and (v) tetrahedral. The particles are different in terms of their shapes (Fig. 1 and Fig. S1, ESI $\dagger$ ) and the number of lobes attached to the seed. To understand the effect of lobe size on self-assembled morphologies, we modeled our particles to closely resemble the experimental method of synthesizing patchy lobed particles, as proposed by Pine and co-workers. ${ }^{11}$ To mimic the experimental protocol for particle design, we first make clusters of spherical particles, and then grow the central repulsive spheres from the centers of mass of these clusters. We term these central repulsive spheres

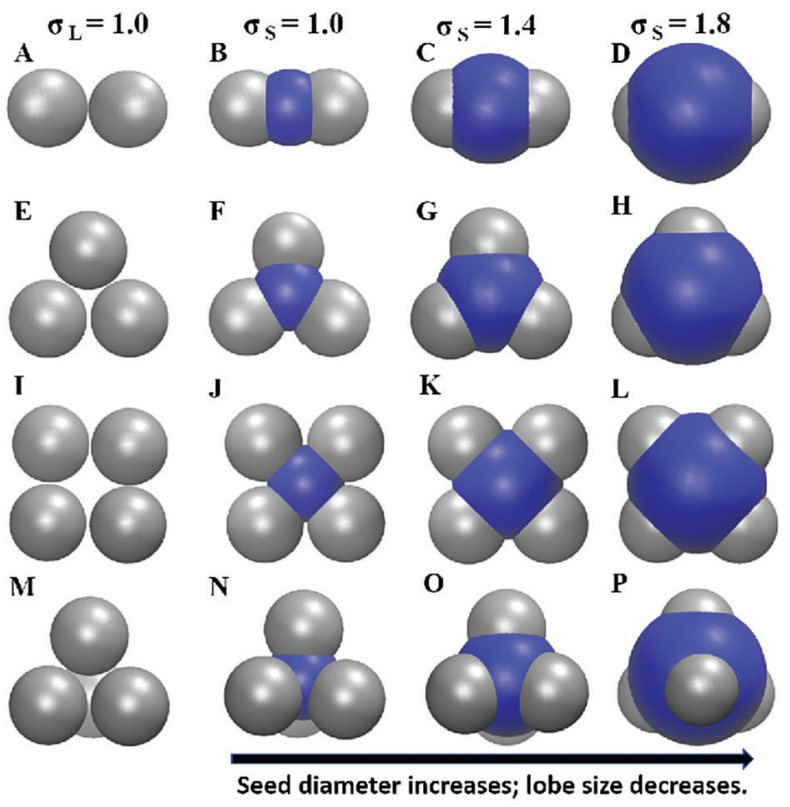

Fig. 1 A pictorial representation of changing the lobe (gray) size by controlling the seed (blue) diameter for dumbbell (A-D), trigonal planar $(E-H)$, square planar $(I-L)$, and tetrahedral $(M-P)$ shaped lobed particles. The clusters of spherical beads of diameter $\sigma_{\mathrm{L}}=1.0$ are shown in the first column (A, E, I and M). The seed diameter was gradually increased from $\sigma_{\mathrm{S}}=1.0$ to 2.0 . We show lobed particles only for three seed diameters $\left(\sigma_{\mathrm{S}}\right)$ : 1.0 (second column), 1.4 (third column), and 1.8 (fourth column). For the snowman shaped particles, we follow a different way to vary the lobe size (Fig. S1, ESI $†$ ).

as seeds in this work complying with our previous work on these lobed particles. ${ }^{28}$ However, from an experimental point of view, seeds are usually referred to as the initial spheres where a second stage reaction is carried out to grow lobes. In the case of tetrahedral lobed particles, for example, we first created a uniform tetrahedral cluster using four spherical beads (Fig. 1M). Then, we placed the seed at the center of mass of the cluster. The exposed parts of spherical beads that remain after inserting the seed become lobes. We gradually increase the diameter of the seed to control the lobe size (Fig. 1N-P). A higher seed diameter leads to a smaller lobe size. We follow the same approach for all lobed particles except for the snowman shaped particles because these particles have only one lobe. Therefore, it is not meaningful to make a cluster using one spherical bead and inserting the seed at the center of mass of the cluster. In that case, we placed a lobe and a seed (two spherical beads) at $0.5 \sigma_{\mathrm{L}}\left(\sigma_{\mathrm{L}}\right.$ is the diameter of the spherical beads and the distance units used in all simulations) apart and varied the seed diameter to obtain different lobe sizes (Fig. S1, ESI $\dagger$ ).

We carried out Langevin molecular dynamics simulations in dimensionless units using the HOOMD-Blue software. ${ }^{34,35}$ The diameters of spherical beads that constitute the initial cluster follow distance units $\left(\sigma_{\mathrm{L}}=1.0\right)$. We gradually increase the seed diameter starting from 1.0 to $2.0\left(\sigma_{\mathrm{S}}=1.0,1.2,1.4,1.6,1.8,2.0\right)$. We define a dimensionless parameter, $q$, which is the ratio of the diameter of spherical beads forming the initial clusters to the diameter of the central seed, $q=\sigma_{\mathrm{L}} / \sigma_{\mathrm{S}}$. As we increase the 
seed diameter from 1.0 to 2.0 , the value of $q$ decreases from 1.0 to $0.5(q=1.0,0.83,0.71,0.63,0.56,0.50)$ with a decrease in the lobe size. The masses of the lobes and seeds are held constant and equal to $1.0\left(m_{\mathrm{S}}=m_{\mathrm{L}}=1.0\right)$.

All types of non-bonded interactions (lobe-lobe, seed-seed, and seed-lobe) between the particles are modeled by a surface shifted Lennard-Jones (SSLJ) potential (see Supplemental methods, ESI $\dagger$ ). We studied the effect of temperature on the selfassembled morphologies and their porosities. We randomized the initial configurations by simulating each system at a higher temperature $\left(k_{\mathrm{B}} T=1.8\right)$ before simulating the self-assembly at other temperatures. We investigated a wide range of temperatures starting from 0.2 to $1.2\left(k_{\mathrm{B}} T=0.2,0.4,0.6,0.8,1.0,1.2\right)$. The inter-lobe interactions employed in this work $\left(\varepsilon_{\mathrm{LL}}=1.0 ; \varepsilon_{\mathrm{LL}}\right.$ is the well depth in the SSLJ potential for the lobe-lobe pairs) vary from $0.83 k_{\mathrm{B}} T$ (at $k_{\mathrm{B}} T=1.2$ ) to $5 k_{\mathrm{B}} T$ (at $k_{\mathrm{B}} T=0.2$ ), which is experimentally realizable. In a recent work, Tsyrenova et al. ${ }^{19}$ reported on how the van der Waals attractions between metal coated patches can be altered by changing the thickness of the metal.

Depending on the number of lobes, the lobe size, and the temperature, we observed that lobed patchy particles selfassemble into different morphologies. We calculated radial distribution functions (RDFs) to understand the morphologies of the self-assembled structures and also to distinguish between different structures generated at different seed diameters and temperatures (see Supplemental methods, ESI $\dagger$ ). We also report the change in the potential energy per particle with time to examine the stability of the self-assembled structures (Fig. S2, ESI $\dagger$ ). Here, we report equilibrium morphologies and porosities obtained from self-assembled structures at the end of each simulation.

\section{Self-assembled morphologies}

Snowman shaped particles show self-assembly only at a very low temperature $\left(k_{\mathrm{B}} T=0.2\right)$ and are observed to generate three different morphologies at three different seed diameters, $\sigma_{\mathrm{S}}=$ $1.0(q=1.0), 1.2(q=0.83)$, and $1.4(q=0.71)$. At $\sigma_{\mathrm{S}}=1.0$, these particles self-assemble into two layers to form two-dimensional sheet like structures (Fig. 2B). In the two layers of these sheets, the lobes remain buried and the seeds remain exposed to the solvent. The RDFs computed for the seed pairs show multiple peaks at a regular interval (Fig. 2A), indicating the presence of highly correlated and ordered particles. The two-dimensional sheets were found to be disassembled at a higher temperature, as we examined its stability by simulating the low-temperature structures at a higher temperature $\left(k_{\mathrm{B}} T=0.4\right)$. When the seed diameter is increased to 1.2 , snowman particles assemble into elongated micelles (Fig. 2C), and into spherical micelles (Fig. 2D) at a seed diameter of 1.4. If the seed diameter is increased beyond 1.4, snowman particles do not self-assemble at any of the temperatures employed in this work. This occurs due to a larger repulsive seed which prevents the particles from approaching closer for self-assembly. The morphologies generated by these particles are similar to an earlier work by Avvisati et al. ${ }^{36}$ By varying a parameter $l\left(l=2 d /\left(\sigma_{\mathrm{S}}+\sigma_{\mathrm{L}}\right) ; d\right.$ is the distance between the seed and the lobe, $\sigma_{\mathrm{S}}$ is the diameter of the seed
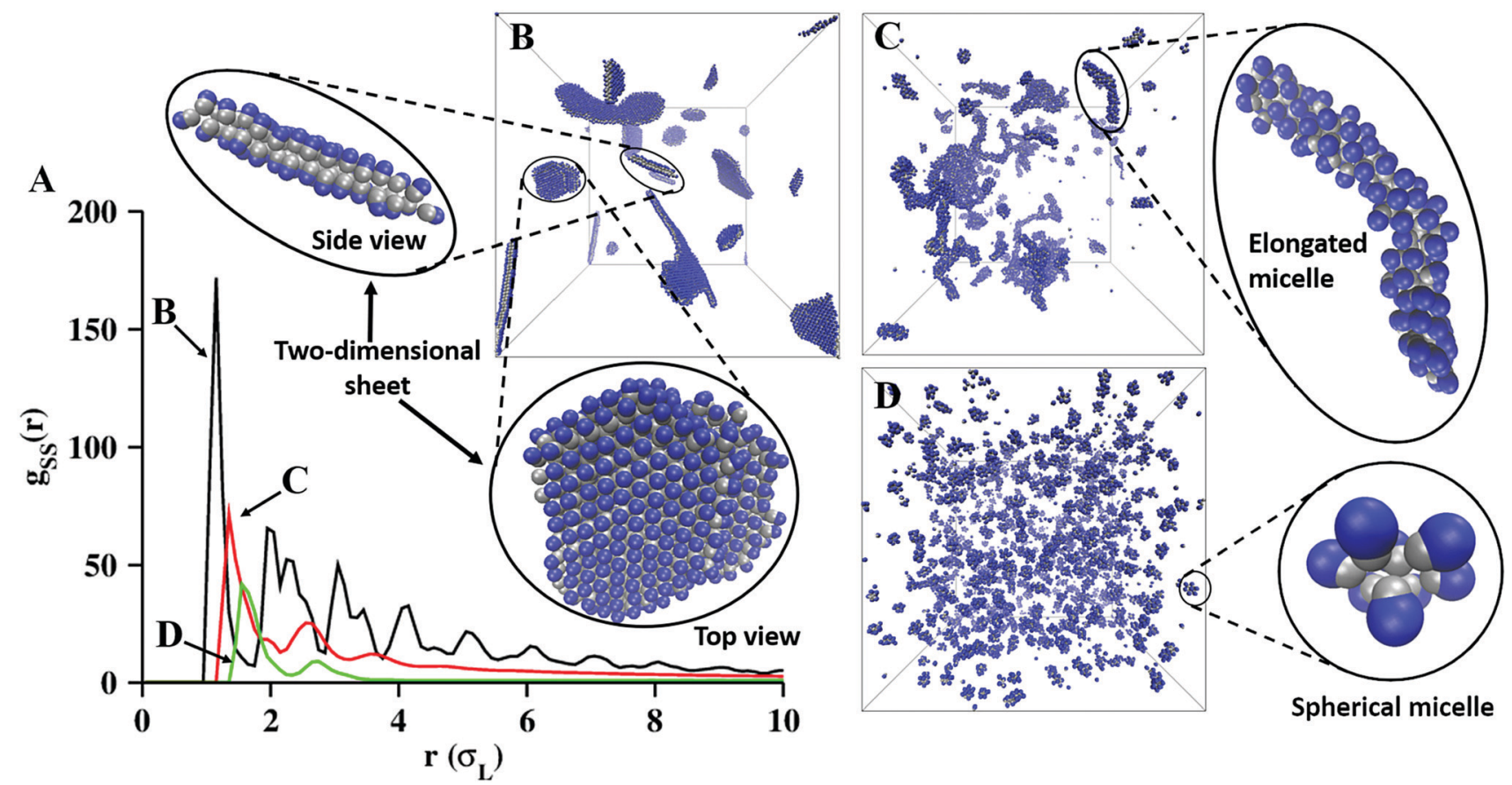

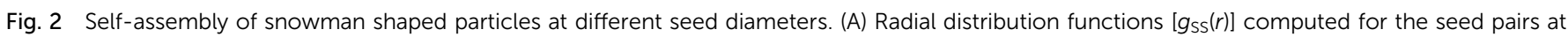

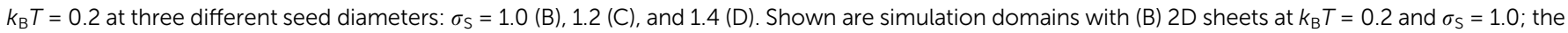

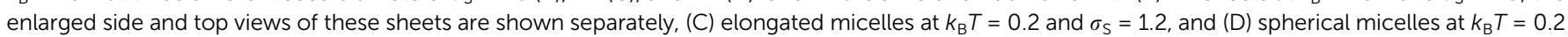
and $\sigma_{\mathrm{S}}=1.4$. 
and $\sigma_{\mathrm{L}}$ is the diameter of the lobe), they showed that snowman shaped particles (referred to as dumbbell shaped particles in their work) self-assemble into sheets at a higher $l$, spherical micelles at a lower $l$, and elongated micelles at a moderate $l$.

At a lower temperature $\left(k_{\mathrm{B}} T=0.2\right)$ dumbbell shaped particles self-assemble into a wide variety of structures at different seed diameters. When the seed diameter is smaller $\left(\sigma_{\mathrm{S}}=1.0\right.$ or $q=1.0$ ) or lobes are larger, these particles self-assemble into random aggregates of cylindrical shapes, as confirmed by the $\mathrm{RDF}$ for the seed-seed pairs where long range order is not present in these aggregates (Fig. 3A and B). When the seed diameter is increased $\left(\sigma_{\mathrm{S}}=1.4\right.$ or $\left.q=0.71\right)$, the random aggregates are likely to attain spherical shapes (Fig. 3C) and the seeds are more ordered, which is confirmed by the corresponding RDF. If the lobes are made very small by increasing the seed size $\left(\sigma_{\mathrm{S}}=1.8\right.$ or $\left.q=0.56\right)$, dumbbell particles selfassemble into crystalline structures (Fig. 3D). We find two different types of unit cells comprising these crystalline morphologies, face centered cubic and body centered cubic. At a higher temperature, dumbbell shaped particles mainly self-assemble into spherical aggregates irrespective of the seed or the lobe size. When the seeds are smaller or lobes are larger, these aggregates are mainly random (Fig. S3A, ESI $\dagger$ ), but if the seed diameter is increased, the aggregates resemble liquid droplets showing that the particles are ordered up to the second or third coordination shells (Fig. S3B, ESI $\dagger$ ).

For trigonal planar, square planar, and tetrahedral lobed particles, we find a similar type of self-assembly behavior. For each type of particle, we identified four distinct self-assembled phases: random aggregates, spherical aggregates, liquid like droplets, and crystalline structures (Fig. 4). At lower temperatures, particles self-assemble into random aggregates for a wide range of seed diameters (green regions in Fig. 4). Random aggregates appear in different shapes (e.g., cylindrical, wire like) and lack correlation between the particles present in them. With an increase in temperature, random aggregates are observed to undergo a transition to spherical aggregates (yellow regions in Fig. 4) at lower seed diameters, and to liquid droplets (blue regions in Fig. 4) at moderate seed diameters. For each type of particle, the transition from random aggregates to liquid droplets happens along two different pathways depending on the seed diameter. At $\sigma_{\mathrm{S}}=1.4(q=0.71)$, this transition is observed to occur via an intermediate phase composed of spherical aggregates, and at $\sigma_{\mathrm{S}}=1.6(q=0.63)$, a direct transition can occur from random aggregates to liquid droplets. At higher seed diameters, when the lobes are very small, interactions between the particles become highly directional, and we observed crystalline assembled structures at lower
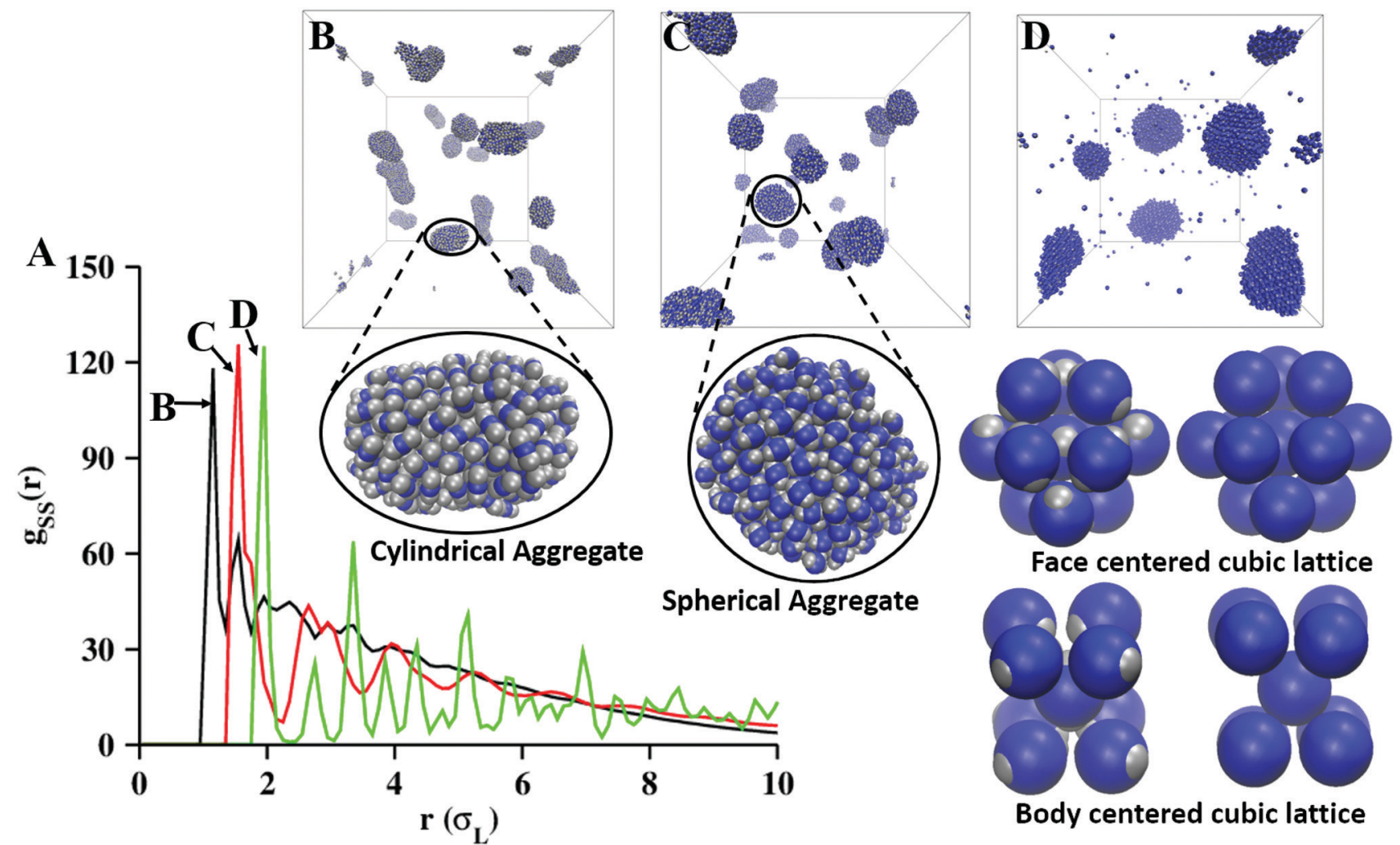

Fig. 3 Self-assembly of dumbbell shaped particles at $k_{\mathrm{B}} T=0.2$. (A) Radial distribution functions $\left[g_{\mathrm{sS}}(r)\right]$ computed for the seed pairs at $k_{\mathrm{B}} T=0.2$ at three different seed diameters; $\sigma_{S}=1.0(q=1.0)(B), 1.4(q=0.71)(C)$, and $1.8(q=0.56)(D)$. Shown are simulation domains with (B) random aggregates of cylindrical shapes at $k_{\mathrm{B}} T=0.2$ and $\sigma_{\mathrm{S}}=1.0$, (C) spherical aggregates at $k_{\mathrm{B}} T=0.2$ and $\sigma_{\mathrm{S}}=1.4$; the enlarged views of the cylindrical and spherical aggregates are shown, and (D) crystalline structures at $k_{\mathrm{B}} T=0.2$ and $\sigma_{\mathrm{S}}=1.8$. Two different unit cells, face centered cubic and body centered cubic (with and without lobes), are also shown. 

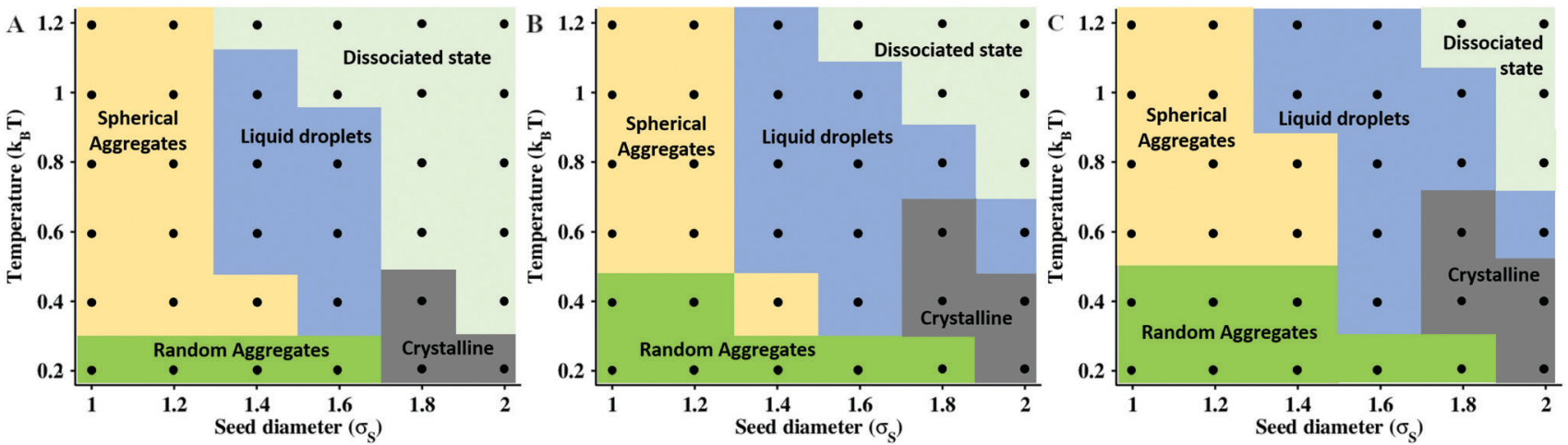

Fig. 4 Phase behavior of (A) trigonal planar, (B) square planar, and (C) tetrahedral lobed particles at different seed diameters and temperatures. The black dots show the exact locations of the seed diameters and temperatures at which the self-assembly studies of the lobed particles were carried out.

temperatures. We computed the number of particles (seeds) present in the first coordination shells of the crystalline structures and found it to be 12 for all three types of lobed particles. For trigonal planar and tetrahedral particles, the unit cell possesses a hexagonal close packed geometry and for square planar particles, it is a face centered cubic. At higher seed diameters and higher temperatures, lobed particles appear in a dissociated state. In the case of trigonal planar particles, crystalline structures directly transitioned to a dissociated state with an increase in temperature, and for square planar and tetrahedral lobed particles, this transition occurs via a liquid droplet phase. However, these transitions are found to occur at different temperatures depending on the number of lobes and shapes of the particles. For example, at $\sigma_{\mathrm{S}}=1.6(q=0.63)$, trigonal planar particles undergo a transition from the dissociated state to a liquid droplet state when temperature is decreased to 0.8 from 1.0. The same transition for the square planar particles at the same seed diameter occurs upon decreasing the temperature from 1.2 to 1.0. For tetrahedral lobed particles, this transition occurs at a temperature higher than 1.2. These differences in the transition temperatures, that arise due to differences in the number of lobes and the shapes of particles, are potentially useful to separate constituent lobed patchy particles from a mixture. The selfassembled morphologies and the corresponding RDFs for these three types of particles are given in the ESI $\dagger$ (Fig. S4-S6).

The morphologies of the self-assembled structures presented so far were obtained at a low number density of particles $\left(\rho_{\mathrm{N}}=0.008\right)$. To understand the effect of particle density on the self-assembled structures, we carried out simulations for all five types of particles at three higher densities $\left(\rho_{\mathrm{N}}=0.016,0.037\right.$, 0.125 ) at a few selected seed diameters and temperatures. These simulations reveal that the self-assembled morphologies are independent of the particle density. For example, snowman shaped particles at $\sigma_{\mathrm{S}}=1.0(q=1.0)$ and at $k_{\mathrm{B}} T=0.2$ form twodimensional sheets at three different higher densities (Fig. S7, ESI $\dagger$ ). As expected, the size of each self-assembled structure is found to increase with an increase in $\rho_{\mathrm{N}}$ (Fig. S7 and S8, ESI $\dagger$ ).

\section{Porosity of self-assembled structures}

We investigated porosities for those cases where larger three dimensional structures are formed through self-assembly. In that aspect, we ruled out the self-assembled structures formed by the snowman shaped particles, as these structures are either two dimensional sheets or micelles (Fig. 2). We computed pore size distributions (PSD) for the self-assembled structures obtained from the other four types of lobed particles using the Zeo++ software. ${ }^{37,38}$ We extracted large cuboids from the assembled structures (Fig. S9, ESI $\dagger$ ) and used those cuboids to determine the PSDs, which are given in the ESI $\dagger$ (Fig. S10-S13). For all types of lobed particles, we observed a similar trend in the variation of PSDs. At lower seed diameters and temperatures, the pore diameters are very small $\left(\sim 0.1 \sigma_{\mathrm{L}}\right)$ in random aggregates, and the pore diameters are found to increase with an increase in temperature. At moderate seed diameters, the distributions are broader at higher temperatures due to the formation of liquid droplets. For example, in the case of dumbbell shaped particles, the pore diameters vary from $0.1 \sigma_{\mathrm{L}}$ to $2.3 \sigma_{\mathrm{L}}$ at $\sigma_{\mathrm{S}}=1.6(q=0.63)$ and $k_{\mathrm{B}} T=0.4$ (Fig. S10D, ESI $\left.\dagger\right)$. For tetrahedral lobed particles, at $\sigma_{\mathrm{S}}=1.6(q=0.63)$ and $k_{\mathrm{B}} T=1.2$, the pore diameters vary from $0.1 \sigma_{\mathrm{L}}$ to $1.6 \sigma_{\mathrm{L}}$ (Fig. S13D, ESI $\dagger$ ). In the crystalline structures self-assembled at higher seed diameters and lower temperatures, the pore diameters are uniform and we observed sharp peaks in the pore size distributions (Fig. S10E and S11F, ESI $\dagger$ ). The pore diameters generated by the lobed particles are comparable to the diameter of the spherical beads $\left(\sigma_{\mathrm{L}}\right)$ that constitute the initial clusters. If we project our dimensionless simulations into real units, and if we choose $1 \sigma_{\mathrm{L}}=1 \mu \mathrm{m}$, then the porosities will vary from $0.1 \mu \mathrm{m}$ to $2.3 \mu \mathrm{m}$, depending on simulation conditions and the particle type. The largest pores, that we found in this study, are produced by the dumbbell shaped particles. The pore diameters for selfassembled structures formed by these particles are $\sim 2.3 \sigma_{\mathrm{L}}$.

In summary, by performing a series of Langevin dynamics simulations, we revealed how two crucial parameters, lobe size and temperature, control the morphologies, phases, and porosities of self-assembled structures generated by five different lobed patchy particles. We have introduced a modeling approach, which mimics a recently developed experimental method of synthesizing patchy particles ${ }^{11}$ to model the lobed particles with different lobe sizes. Snowman shaped particles, which self-assemble only at a lower temperature, formed two-dimensional sheets, elongated and spherical micelles depending on the lobe size. Each of the 
other four types of particles (dumbbell, trigonal planar, square planar, and tetrahedral) was found to self-assemble into four distinct morphologies (random aggregates, spherical aggregates, liquid droplets, and crystalline) depending on the lobe size and temperature. At higher seed diameters (smaller lobes) and higher temperatures, these particles do not self-assemble and remain in a dissociated state. The self-assembled morphologies were found to be the same at a range of particle densities. The transitions between any two specific phases occur at different temperatures for different lobed particles. This difference in the transition temperature (mainly for the transition from a dissociated state to a condensed phase) could be useful in separating a mixture of different types of lobed particles. The self-assembled structures generated by these four types of particles are porous and their porosities are found to depend on the lobe size and temperature. The pore diameters increase with an increase in temperature at any particular seed diameter. The largest pores were produced by the dumbbell shaped particles. We suggest that the porosities of the self-assembled structures may be increased by increasing the repulsive interactions between seeds, possibly by including electrostatic interactions in future models.

\section{Conflicts of interest}

There are no conflicts to declare.

\section{Acknowledgements}

We are grateful for financial support provided by the National Science Foundation (award no. OIA-1757371; HV). We acknowledge computational support through the following resources: Premise, a central shared HPC cluster at UNH supported by the Research Computing Center; BioMade, a heterogeneous CPU/ GPU cluster supported by the NSF EPSCoR award (OIA-1757371; $\mathrm{HV}$ ); and the NSF-supported (ACI-1548562) Extreme Science and Engineering Discovery Environment (XSEDE) ${ }^{39}$ Comet resource at San Diego Supercomputer Center (SDSC) under grant TG-MCB160183 (HV).

\section{Notes and references}

1 S. Ravaine and E. Duguet, Curr. Opin. Colloid Interface Sci., 2017, 30, 45-53.

2 F. Li, D. P. Josephson and A. Stein, Angew. Chem., Int. Ed., 2011, 50, 360-388.

3 S. C. Glotzer, M. J. Solomon and N. A. Kotov, AIChE J., 2004, 50, 2978-2985.

4 Y. Xia, B. Gates and Z.-Y. Li, Adv. Mater., 2001, 13, 409-413.

5 A. B. Pawar and I. Kretzschmar, Macromol. Rapid Commun., 2010, 31, 150-168.

$6 \mathrm{M}$. Boncheva and G. M. Whitesides, in Biomimetic Approaches to the Design of Functional, Self-Assembling Systems, ed. J. A. Schwarz, C. I. Contescu and K. Putyera, Marcel Dekker, Inc., New York, NY, 1st edn, 2004, vol. 1, pp. 287-294.

7 Z. Zhang and S. C. Glotzer, Nano Lett., 2004, 4, 1407-1413.
8 Z. Zhang, A. S. Keys, T. Chen and S. C. Glotzer, Langmuir, 2005, 21, 11547-11551.

9 Q. Chen, S. C. Bae and S. Granick, Nature, 2011, 469, 381-384.

10 D. J. Kraft, R. Ni, F. Smallenburg, M. Hermes, K. Yoon, D. A. Weitz, A. van Blaaderen, J. Groenewold, M. Dijkstra and W. K. Kegel, Proc. Natl. Acad. Sci. U. S. A., 2012, 109, 10787-10792.

11 Y. Wang, Y. Wang, D. R. Breed, V. N. Manoharan, L. Feng, A. D. Hollingsworth, M. Weck and D. J. Pine, Nature, 2012, 491, 51-55.

12 K. H. Ku, Y. Kim, G.-R. Yi, Y. S. Jung and B. J. Kim, ACS Nano, 2015, 9, 11333-11341.

13 J. A. Diaz A., J. S. Oh, G.-R. Yi and D. J. Pine, Proc. Natl. Acad. Sci. U. S. A., 2020, 117, 10645-10653.

14 I. C. Pons-Siepermann and S. C. Glotzer, ACS Nano, 2012, 6, 3919-3924.

15 A. W. Long and A. L. Ferguson, Mol. Syst. Des. Eng., 2018, 3, 49-65. 16 A. W. Long and A. L. Ferguson, J. Phys. Chem. B, 2014, 118, 4228-4244.

17 D. Morphew, J. Shaw, C. Avins and D. Chakrabarti, ACS Nano, 2018, 12, 2355-2364.

18 T. S. Skelhon, Y. Chen and S. A. F. Bon, Soft Matter, 2014, 10, 7730-7735.

19 A. Tsyrenova, K. Miller, J. Yan, E. Olson, S. M. Anthony and S. Jiang, Langmuir, 2019, 35, 6106-6111.

20 D. J. Kraft, W. S. Vlug, C. M. van Kats, A. van Blaaderen, A. Imhof and W. K. Kegel, J. Am. Chem. Soc., 2009, 131, 1182-1186.

21 L. Hong, A. Cacciuto, E. Luijten and S. Granick, Langmuir, 2008, 24, 621-625.

22 B. Bharti, D. Rutkowski, K. Han, A. U. Kumar, C. K. Hall and O. D. Velev, J. Am. Chem. Soc., 2016, 138, 14948-14953.

23 J. S. Oh, S. Lee, S. C. Glotzer, Y. Gi-Ra and D. J. Pine, Nat. Commun., 2019, 10, 3936.

24 A. W. Wilber, J. P. K. Doye and A. A. Louis, J. Chem. Phys., 2009, 131, 175101.

25 A. Giacometti, F. Lado, J. Largo, G. Pastore and F. Sciortino, J. Chem. Phys., 2010, 132, 174110.

26 F. Liu, S. Goyal, M. Forrester, T. Ma, K. Miller, Y. Mansoorieh, J. Henjum, L. Zhou, E. Cochran and S. Jiang, Nano Lett., 2019, 19, 1587-1594.

27 V. N. Manoharan, M. T. Elsesser and D. J. Pine, Science, 2003, 301, 483-487.

28 S. Paul and H. Vashisth, Soft Matter, 2020, 16, 1142-1147. 29 Q. L. Loh and C. Choong, Tissue Eng., Part B, 2013, 19, 485-502. 30 J. B. Matson, R. H. Zha and S. I. Stupp, Curr. Opin. Solid State Mater. Sci., 2011, 15, 225-235.

31 S. Sacanna, M. Korpics, K. Rodriguez, L. Colón-Meléndez, S.-H. Kim, D. J. Pine and G.-R. Yi, Nat. Commun., 2013, 4, 1688.

32 Z. Gong, T. Hueckel, G.-R. Yi and S. Sacanna, Nature, 2017, 550, 234-238.

33 V. Meester, R. W. Verweij, C. van der Wel and D. J. Kraft, ACS Nano, 2016, 10, 4322-4329.

34 J. A. Anderson, C. D. Lorenz and A. Travesset, J. Comput. Phys., 2008, 227, 5342-5359. 
35 J. Glaser, T. D. Nguyen, J. A. Anderson, P. Lui, F. Spiga, J. A. Millan, D. C. Morse and S. C. Glotzer, Comput. Phys. Commun., 2015, 192, 97-107.

36 G. Avvisati, T. Vissers and M. Dijkstra, J. Chem. Phys., 2015, $142,084905$.

37 M. Pinheiro, R. L. Martin, C. H. Rycroft and M. Haranczyk, CrystEngComm, 2013, 15, 7531-7538.
38 M. Pinheiro, R. L. Martin, C. H. Rycroft, A. Jones, E. Iglesia and M. Haranczyk, J. Mol. Graphics Modell., 2013, 44, 208-219.

39 J. Towns, T. Cockerill, M. Dahan, I. Foster, K. Gaither, A. Grimshaw, V. Hazlewood, S. Lathrop, D. Lifka, G. D. Peterson, R. Roskies, J. R. Scott and N. WilkinsDiehr, Comput. Sci. Eng., 2014, 16, 62-74. 